\title{
Ovarian hemangioma associated with tamoxifen therapy: a case report
}

\author{
Salvatore Lanzafame $\cdot$ Rosario Caltabiano • \\ Antonio Giuseppe Nicolosi $\cdot$ Santi Palmeri
}

Received: 16 March 2007 / Accepted: 4 July 2007 / Published online: 15 September 2007

(C) Springer-Verlag 2007

\begin{abstract}
Vascular tumors of the ovary are very rare. We report a case of ovarian hemangioma in a patient treated with tamoxifen for breast ductal carcinoma. CD31 and CD34 immunoreactivity confirmed the vascular origin of the tumor. It is interesting to note that estrogen and progesterone receptors were negative in endothelial cells of the hemangioma, but were positive in stromal ovarian cells. Tamoxifen is a synthetic, non-steroidal, anti-estrogenic drug widely used as adjuvant therapy for pre- and postmenopausal, early and metastatic, breast cancer patients with positive estrogen receptor proteins. The mechanism of action of tamoxifen in stimulating the development and/ or growth of ovarian hemangioma is unknown. We may speculate that its prolonged, estrogenic effect on the ovary may be one of the stimulating factor.
\end{abstract}

Keywords ovarian hemangioma $\cdot$ tamoxifen $\cdot$ therapy

\section{Introduction}

Vascular tumors of the ovary are very rare. This is considered to be unusual in view of the rich vascular supply of the ovary. Ovarian hemangiomas may occur in association with generalized hemangiomatosis or as an

S. Lanzafame $(\bowtie) \cdot R$. Caltabiano

Department G.F. Ingrassia, Section of Anatomic Pathology,

University of Catania,

Santa Sofia 87 street,

95123 Catania, Italy

e-mail: lanzafas@unict.it

A. G. Nicolosi $\cdot$ S. Palmeri

U.O. Casa di Cura Basile, Oncologic Surgery,

Catania, Italy isolated neoplasm. While in the first case, patients have an high risk of intravascular coagulation and Kasabach-Merritt syndrome [1], isolated hemangiomas are usually incidental findings due to their small size and asymptomatic nature. We report a case of ovarian hemangioma in a patient treated with tamoxifen for breast ductal carcinoma.

\section{Case report}

A 66-year-old woman presented with a right adnexal mass and abdominal pain. Her last menstrual period occured 17 years previously, and she had no abnormal uterine bleeding. Patient's past medical history revealed in 2002 an hypoechoic cyst in the same ovary measuring $55 \mathrm{~mm}$ (Fig. 1) and a breast invasive ductal carcinoma, with a marked nuclear immunoreactivity for estrogen and progesterone receptors, treated with tamoxifen. In ultrasound scans performed until 3 years later, the ovarian cyst kept the same echogenic aspect. In 2006, after 4 years' treatment with tamoxifen, ultrasound examination revealed an enlarged, hyperechoic, right ovary measuring $6 \times 4 \mathrm{~cm}$, suspected for breast metastasis (Fig. 1). Computer tomography (CT) scanning of the abdomen and pelvis demonstrated a 6-cm complex mass with both cystic and solid components of ovarian origin (Fig. 2). Complete blood counts and serum hormone levels were within normal limits. An endometrial biopsy showed normal mucosa. At laparotomy, the right ovary was dark, red and enlarged, measuring $6 \times 4 \times 3 \mathrm{~cm}$. The left ovary and the uterus were unremarkable. There was no palpable pelvic or periaortic lymphadenopathy. Intraoperative microscopic examination of the right ovary was negative for malignant neoplasm. Therefore, an unilateral oophorectomy was performed. At gross examination, the cut surface of the resected ovary was 
Fig. 1 Patient's past medical history revealed an hypoechoic cyst in the right ovary (on the left). After 4 years therapy with tamoxifen, ultrasound examination revealed an enlarged, hyperechoic, right ovary (on the right)

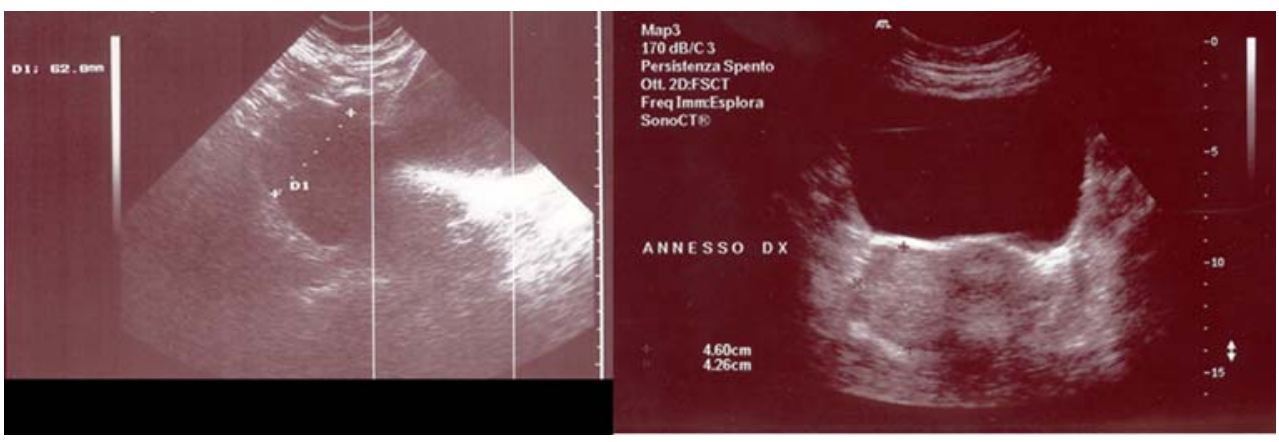

spongy and had a honeycomb appearance (Fig. 3). Histological examination demonstrated multiple, enlarged, blood-filled vascular channels lined by a single layer of flattened regular endothelial cells with no atypical features. Vascular spaces within the tumor were of different sizes, ranging from small to large, and were separated by cellular connective tissue. The histological diagnosis was ovarian cavernous hemangioma. CD31 and CD34 immunoreactivity confirmed the vascular origin of the tumor. It is interesting to note that estrogen and progesterone receptors were negative in endothelial cells of the hemangioma, but were positive in stromal ovarian cells (Fig. 4). Tamoxifen therapy was stopped after the operation.

\section{Discussion}

Tamoxifen is a synthetic, non-steroidal, anti-estrogenic drug widely used as adjuvant therapy for pre- and postmenopausal, early and metastatic, breast cancer patients with positive estrogen receptor proteins. A combination of tamoxifen and chemotherapy reduces the risk recurrence as well as the incidence of controlateral breast cancer.

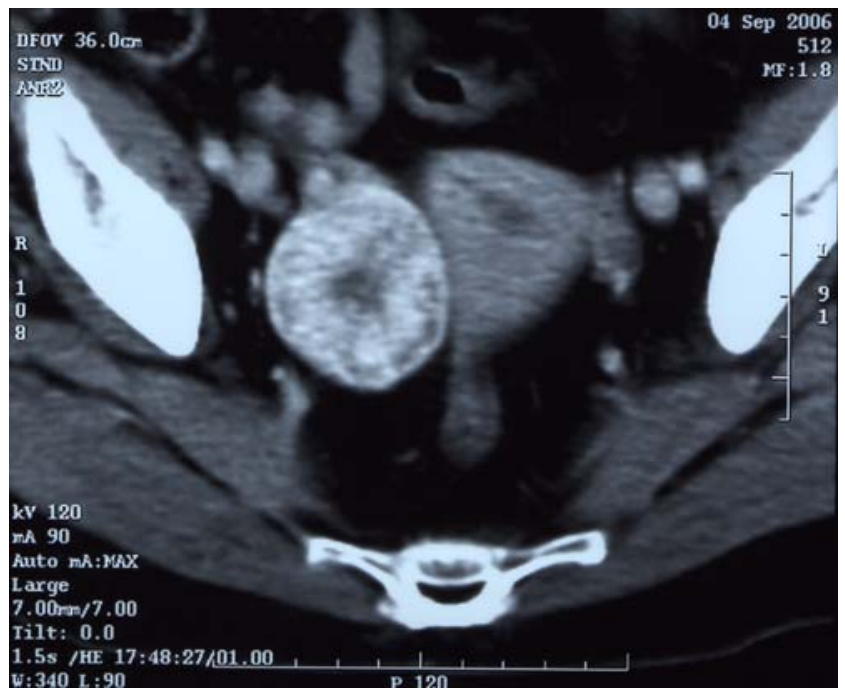

Fig. 2 Computer tomography (CT) scanning of the abdomen and pelvis demonstrated a complex mass with both cystic and solid components of ovarian origin
Tamoxifen decreases the incidence of primary breast cancer in high risk healthy women [2]. It acts as a selective estrogen receptor modulator and has different side effects in different tissues. The antiestrogenic activity may be related to its ability to inhibit the binding of estradiol to the estrogen receptor or to decrease estrogen receptor levels [3]. Although tamoxifen acts primarily as an antiestrogen, it also has a mild estrogenic effect. In fact, it has been found to be associated with various uterine pathologies such as adenomyosis, hyperplasia, endometrial polyps, endometrial carcinoma and endometrial sarcoma [4]. On the ovary, tamoxifen induces steroidogenesis in pre-menopausal women and induces ovulation. Several recently published case reports suggest that there might be a link between tamoxifen therapy and ovarian cysts [5] or even an increased risk of ovarian cancer [6]. Nevertheless, the link between tamoxifen and ovarian cancer in premenopausal women is controversial, and other studies suggest that tamoxifen treatment of premenopausal or perimenopausal women does not materially affect ovarian cancer risk [7]. The mechanism of action of tamoxifen in stimulating the development of ovarian cysts has not been clearly explained. Considering that premenopausal breast cancer patients produce high amounts of estrogen, it was suggested that tamoxifen induces ovarian cysts in premenopausal

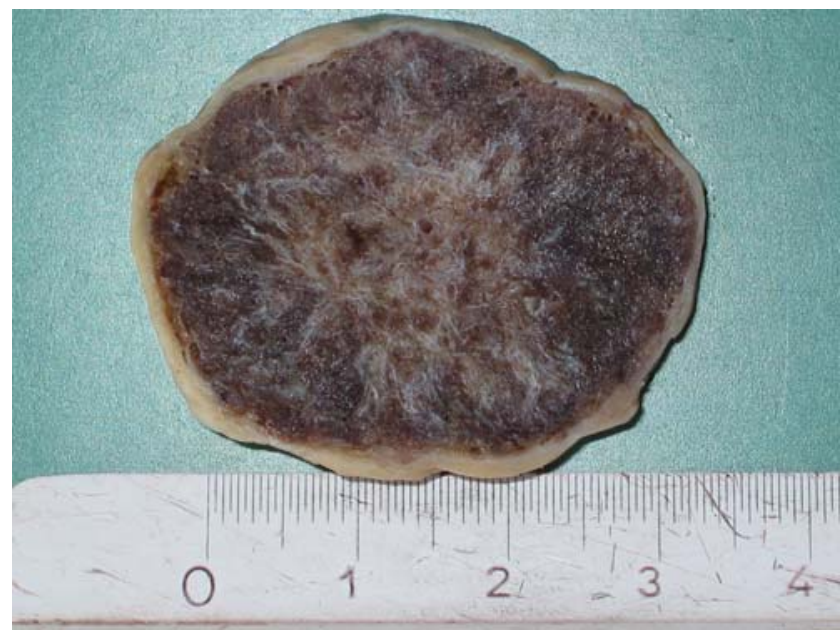

Fig. 3 At gross examination, the cut surface of the resected ovary was spongy and had a honeycomb appearance 
Fig. 4 Estrogen receptors are negative in endothelial cells of the hemangioma, but positive in stromal ovarian cells (10X) (on the left). Progesterone receptors are negative in endothelial cells of the hemangioma, but positive in stromal ovarian cells $(10 \mathrm{X})$ (on the right)
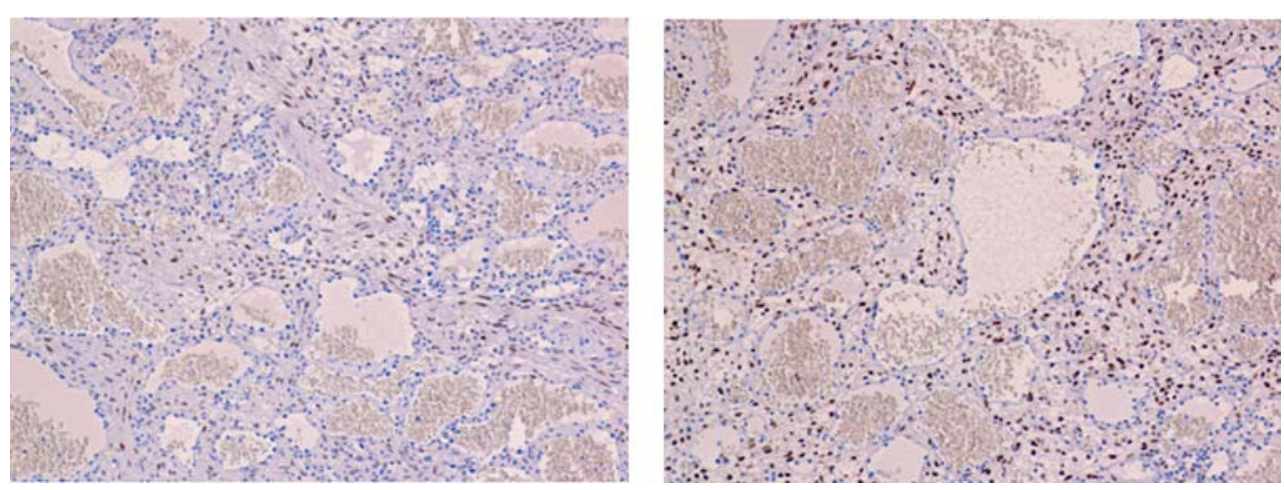

women with its direct action on the ovaries to stimulate excessive growth of ovarian follicles, resulting in elevated estradiol levels. Such a direct action of tamoxifen on the ovaries may be mediated by the activity of insulin-like growth factor-I in granulosa cells, similar to the direct effect of the tamoxifen on the endometrium [8]. Tamoxifen, however, does not change the serum estradiol level in postmenopausal women. In such cases it might have a direct estrogenic effect on the ovaries similar to the direct effect on the endometrium of post-menopausal women, increasing estradiol receptor levels [3]. The enlargement of the ovaries in women treated with tamoxifen, can result from functional cysts, metastases of the primary breast cancer, or primary ovarian malignancy (women with breast cancer have an increased risk for ovarian cancer). In such cases, patients must be surgically explored to enable pathologic examination and exclusion of malignancy. The occurrence of ovarian hemangioma in a patient treated with tamoxifen has never been reported in the literature. Ovarian hemangiomas are very rare and histologically may be cavernous or capillary with the first type being the most common [1]. The difference between them relates to the size of the blood vessels formed. Capillary hemangiomas are made up of vessels of the caliber of normal capillaries, while the cavernous hemangiomas consist of larger channels. Most cases of ovarian hemangiomas are small and are incidental findings at operation or autopsy. Large lesions tend to present clinically with abdominal mass and/or pain, acute abdomen, ascites. The mechanism of action of tamoxifen in stimulating the development and/or growth of this lesion is unknown. We may speculate that its prolonged, estrogenic effect on the ovary may be one of the stimulating factor. As reported in the literature there is evidence to support a role for estrogens in influencing ovarian development from early fetal life. Estrogen action, like that of other steroids, is mediated by specific receptors in target cells, which on ligand binding undergo a conformational change that results in dimerization and subsequent association with specific regions within the regulatory regions of target genes. A paracrine interaction occurs between estradiol and estrogen receptor to influence the stroma and vascular network in the developing ovary. Some authors have proposed that ovarian hemangiomas are congenital malformations, which may be stimulated by hormonal influences [9]; however in our case endothelial cells were negative for estrogen and progesterone receptors. The high expression of estrogen receptors in stromal cells suggests an indirect effect of estrogens on the hemangioma growth rather than development. In fact, during the 4 years' therapy, the ovary grossly maintained its dimension, but changed the echogenic aspect from a cyst to an hyperechoic mass. Therefore, in our case, tamoxifen probably induced the growth of a previously present little hemangioma undetectable with ultrasound examination. The estrogen-induced stromal signaling that provokes endothelial proliferation is unknown, but it is likely to involve growth factors released by stromal cells [10]. Thus, estrogens may induce the growth of ovarian hemangioma indirectly by regulating the stromal cell-endothelial cell interaction as well as through direct regulation of endothelial cells. In conclusion, no certain evidence regarding a potential link between tamoxifen and ovarian hemangioma exists, and further studies are necessary to obtain conclusions.

\section{References}

1. Uppal S, Heller DS, Majmudar B (2004) Ovarian hemangiomareport of three cases and review of the literature. Arch Gynecol Obstet 270:1-5

2. Fisher B, Dignam J, Bryant J, DeCillis A, Wickerham DL, Wolmark N, Costantino J, Redmond C, Fisher ER, Bowamn DM, Deschenes L, Dimitrov NV, Margolese RG, Robidoux A, Shibata H, Terz J, Paterson AH, Feldman MI, Farrar W, Evans J, Lickley HL (1996) Five versus more than five years of tamoxifen therapy for breast cancer patients with negative lymph nodes and estrogen receptor-positive tumors. J Natl Cancer Inst 88:1529-1542

3. Gorodeski GI, Beery R, Geier A (1992) Tamoxifen increases plasma estrogen-binding equivalents and has an estradiol agonistic effect on histologically normal pre-menopausal and post-menopausal endometrium. Fertil Steril 57:320-327

4. Hardell L (1988) Tamoxifen as risk factor for carcinoma of corpus uteri. Lancet 3 2(8610):563

5. Cohen I, Rosen DJ, Altaras M, Beyth Y, Shapira J, Yigael D (1994) Tamoxifen treatment in premenopausal breast cancer 
patients may be associated with ovarian overstimulation, cystic formations and fibroid overgrowth. Br J Cancer 69:620-621

6. Spicer DV, Pike MC, Henderson BE (1991) Ovarian cancer and long-term tamoxifen in premenopausal women. Lancet 8337 (8754): 1414

7. Swerdlow AJ, Jones ME (2007) Ovarian cancer risk in premenopausal and perimenopausal women treated with Tamoxifen: a case-control study. Br J Cancer 96:850-855
8. Terada S, Uchide K, Suzuki N, Akasofu K (1993) A follicular cyst during tamoxifen therapy in a premenopausal breast cancer woman. Gynecol Obstet Investig 35:62-64

9. DiOrio Jr J, Lowe LC (1980) Hemangioma of the ovary in pregnancy: a case report. J Reprod Med 24:232-234

10. Xiao X, Liu J, Sheng M (2004) Synergistic effect of estrogen and VEGF on the proliferation of hemangioma vascular endothelial cells. J Pediatr Surg 39:1107-1110 